\title{
Current Management of Temporomandibular Joint (TMJ) Disease
}

\author{
Sidebottom $\mathrm{AJ}^{1^{*}}$, Shahme Farook ${ }^{2}$ and Cascarini $\mathrm{L}^{3}$ \\ ${ }^{1}$ Department of Oral and Maxillofacial Surgery, Queen's Medical Centre, Derby Rd, Nottingham, NG72UH, UK \\ ${ }^{2}$ Department of Oral and Maxillofacial Surgery, Northwick Park Hospital, Watford Road, Harrow, Middlesex, HA1 3UJ, UK \\ ${ }^{3}$ Department of Head and Neck Surgery, Guy's Hospital, Great Maze Pond, London SE1 9RT, UK \\ Received: April 22, 2014, Accepted: July 22, 2014, Published: July 30, 2014
}

"Corresponding author: Dr. Sidebottom AJ, Department of Oral and Maxillofacial Surgery, Queen's Medical Centre, Derby Rd, Nottingham, NG7 2UH, UK, Tel: 441159249924 extn: 65895; E-mail: ajsidebottom@doctors.org.uk

Copyright: (c) 2014 Sidebottom AJ, et al. This is an open-access article distributed under the terms of the Creative Commons Attribution License, which permits unrestricted use, distribution, and reproduction in any medium, provided the original author and source are credited.

\begin{abstract}
Temporomandibular joint (TMJ) disorder (TMD) is a collection of medical and dental conditions affecting the joint and muscles of mastication, as well as contiguous tissue components. This leads to pain and altered oral function and can lead to a poor quality of life. The majority of the population can be affected to some degree. Management of TMD is often simple in the first instance, but may involve complex decision making. This article summarizes the current investigative and treatment options available.

Magnetic Resonance Imaging is the recommended radiological investigation of choice for soft tissue assessment whilst TMJ arthroscopy supersedes most other invasive treatments available in relation management of symptoms and more accurate diagnosis. Should arthroscopy fail to achieve satisfactory resolution of symptoms a standardised treatment pathway based on arthroscopic findings involves appropriate assessment, possible open surgery or even TMJ replacement. This latter procedure is governed by NICE guidelines and delivers an excellent short and medium term outcome up to 20 years.
\end{abstract}

Keywords: Temporomandibular joint; Ginglymoid synovial joint; Replacement TMJ pain; Temporomandibular disorder; TMJ arthroscopy

\section{Introduction}

The temporomandibular joint (TMJ) is a load bearing modified bilateral, diarthrodial, ginglymoid synovial joint and permits movements in all three planes. Disruption to the anatomy of this joint from inflammatory changes and trauma could lead to a poor quality of life mainly through pain and functional deficit. TMJ disorder (TMD) is an umbrella term which encompasses pain and dysfunction of the muscles of mastication and TMJ. Over time these symptoms could translate into chronic conditions leading to intractable pain and limitations in jaw function. National Institutes of Health Technology Assessment Conference on Managing Temporomandibular Disorders defined TMJD as "a collection of medical and dental conditions affecting the joint and muscles of mastication, as well as contiguous tissue components" [1].

It is relatively common with a prevalence of $16-59 \%$ reporting symptoms and 33-86\% exhibiting clinical signs. Twenty-five percent of these individuals will proceed to seek treatment [2]. Therefore it is not surprising that TMD is commonly encountered by both medical and dental professionals alike. Maxillofacial surgeons have the access to variety of investigative and treatment modalities but it is paramount to understand and be aware of the current practices of management of the TMJ and the degree of variability in the final outcomes. This article provides a brief overview of current practice and the developments within the management of TMJ disease.

\section{Diagnostic Methods}

Thorough clinical examination helps to localise and determine whether the origin of pain is primarily joint or muscle related. Tenderness over the masticatory muscles would indicate myofascial pain which can be diagnosed by the palpation of trigger points (tense tender bands of muscle). Studies have identified muscle palpation in the diagnosis of TMJD to be less reliable (kappa index 0.47) [3]. Joint pain could be triggered by the nerve endings in the capsular ligaments and retrodiscal tissues and could represent an underlying intraarticular pathology. Deviation of the opening path usually represents either problem with disc mobility, elongation of the coronoid process or ankylosis if lateral deviation is less than $8 \mathrm{~mm}$ [3]. The deviation tends to be towards the side of the pathology and is usually due to intra-articular problems.

Although panoramic radiography is commonly used as a screening tool in patients with TMD, doubts have been raised regarding the reliability in evaluating condylar morphology. Even though it may be suitable for assessing gross bony changes of the condyle; the head position can affect the images of the condyle and fossa significantly. This can lead to anterior condylar flattening, directing towards a misdiagnosis of osteoarthritis [4]. Lack of correlation between panoramic radiography and clinical findings makes this a weak radiological investigation in the management TMD [5]. It is however useful to exclude dental causes for pain and restricted mouth opening.

Cone Beam Computed Tomography (CBCT) is a suitable investigative tool to examine the TMJ without superimposition and distortion to determine the bone morphology, joint space and dynamic function. CBCT provides high-resolution multiplanar images and emits a significantly lower radiation dose compared with CT. CBCT machines are less bulky and available in the community, 
whereas multiplanar CT is only available in a hospital environment. CT provides accurate hard tissue information but poor soft tissue visualisation [6].

$\mathrm{CT}$ as a diagnostic imaging tool for TMD has a reported sensitivity of $75 \%$ and a specificity of $100 \%$ for detecting bony changes [7]. Although the radiation dose involved in the imaging process is a concern, wide accessibility and better tolerance among patients makes it a diagnostic tool which should not be dismissed. In patients where Magnetic Resonance Imaging (MRI) is contraindicated, Multidetector CT (MDCT) remains the next suitable investigation of choice. MDCT have been successfully used to investigate and identify internal disc derangement, arthritis and other miscellaneous other conditions of the TMJ [8].

MRI remains the gold standard investigation to elicit TMJ abnormalities. Internal derangement related to disc displacement is a common MRI finding in patients with TMJ disorder and is present in $80 \%$ of patients consecutively referred for TMJ imaging. Disc displacement, joint effusion, mandibular condyle and marrow abnormalities can be better assessed using MRI [9]. MRI morphological manifestations of TMJ are shown to correlate well with symptoms and confirms the value of MRI in the diagnosis of TMD [10]. However, studies have shown that the diagnostic accuracy of MRI in relation to disc tear is only around 50\% compared with surgical findings at arthroscopy or open surgery [11].

\section{Conservative Management}

Management of TMD involves a conservative and a surgical arm. Rest, occlusal support, non-steroidal anti-inflammatory drugs (NSAID) in combination can be used to treat majority of patients. Occlusal splints of all types have shown to reduce TMJ pain but the results are not significant $[12,13]$. These should cover the whole of the dental arch as localised devices can cause otrthodontic tooth movements. Cochrane assessment shows no evidence for the use of occlusal modification in TMD $[14,15]$. Use of medications to combat symptoms of pain does not manage the underlying disorder [16]. Therapeutic exercises as a non-harmful adjunctive treatment is considered effective in the management of purely myofascial pain. The evidence for this is based on clinical experience rather than clinical trials [17]. It is important in all management techniques to "First do no harm" so irreversible changes which are not supported by adequate literature such as orthodontics, occlusal management, extractions etc. should be avoided.

Pain over the TMJ indicates joint involvement and can be confirmed by injecting local anaesthetic into the joint which should relieve the pain. Arthrocentesis involves the lavage of upper joint space and is minimally invasive. The TMJ is flushed under pressure to reduce inflammatory mediators, release any adhesions and improve joint mobility and pain. Studies have confirmed significant reduction in pain and mouth opening at 1-year follow-up after TMJ arthrocentesis thus concluding it to be a simple, minimally invasive procedure with a relatively low risk of complications and significant clinical benefits [18].

Currently there is no strong evidence to support the benefits of injecting corticosteroids to manage TMD [19,20]; but recent studies have shown some promise with Hyaluronic acid [21,22], although this is by no means conclusive. Myofascial pain may be managed by the injection of botulinum toxin into areas of muscle spasm [23]. Amitriptyline and gabapentin have also been shown to improve pain in patients with myofascial pain but should be used in conjunction with a multidisciplinary pain team [24].

Proliferation treatment also known prolotherapy is regenerative injection therapy practiced since 1937 . Non-pharmacological solutions such as dextrose, psyllium seed oil, phenol and glycerin is injected into the TMJ region. In theory prolotherapy initiates a non-inflammatory or inflammatory process which leads to deposition of additional fibres that will strengthen ligaments and possibly promote the release of local growth factors [25,26]. Studies have confirmed improvement in symptoms of pain and dislocation following prolotherapy, especially in patients who have had conservative treatment with intraoral appliances and physical therapy [27]. This is weak evidence and UK trials are currently under way.

\section{Surgical Management}

\section{Arthroscopy}

TMJ arthroscopy is widely used in the diagnostic and treatment of joint related TMD. This is a low risk procedure with significant benefits which also provides diagnostic support if required for ongoing management. It may be performed either under local or general anaesthesia and usually requires a short post-operative stay. Availability of small, $1.2 \mathrm{~mm}$ arthroscopes has shown maintained outcomes and diagnostic ability compared with larger scopes which potentially cause more morbidity [28]. Restricted view of the lower joint space and operator dependant results can be a drawback of this procedure. Since the majority of cases with restricted mouth opening is due to involvement of the upper joint space, TMJ arthroscopy is invaluable in visualising this space prior to any further management. Arthroscopy and arthrocentesis have shown therapeutic success in reducing pain whilst improving the functional outcome in up to $90 \%$ of cases but no significant therapeutic difference was noted between these two modalities of treatment $[29,30]$. Cochrane review of arthroscopy for TMJ disorders identifies a reduction in pain after 6 months but in comparison to open surgery it was more effective after 12 months. No difference was noted in mandibular function [31].

\section{Open Joint Surgery}

Similar results have been reported following open and arthroscopic joint surgery for internal derangement of the TMJ and hence initial arthroscopy which is minimally invasive should be the preferred choice [32]. Despite this open joint surgery is still advocated for certain TMJ conditions. Eminectomy has been used for the treatment of recurrent dislocations and disc displacement with associated joint pain with favourable results but lacks evidence to support positive outcomes for management of TMJ pain [33]. Other forms of open joint surgery (disc plication, discectomy and condylar shave) have their exponents, all suggesting $80 \%$ benefit following surgery, however recent reports suggest that outcomes are not as good in patients where prior arthroscopy has failed suggesting that some of the good results from open surgery relate to those cases where arthroscopy alone would have been beneficial [34]. Condylar shave is a suitable option to treat condylar hyperplasia and remodelling of joint surface for degenerative progress in internal derangement but there are no long term outcome studies [14].

Discectomy is indicated for the grossly damaged discs which are not salvageable. This is a good choice of treatment for patients with reciprocal clicking or chronic closed lock due to disc disease [35], but 
Page 3 of 4

has poorer outcomes when prior arthroscopy has failed [36]. Although several interpositional materials have been tried, an ideal replacement following TMJ discectomy has not been determined [36]. Whether the disc should be replaced at all is debatable. If these treatment options fail to deliver a good quality of life for the patients and further surgery is indicated there is little to offer other than total joint replacement.

Despite the concept of total joint replacement originating in the 19th century it has only been during the last three decades that TMJ replacement has been given serious consideration due to the long term success of Christensen prosthesis. Currently there are three prostheses available in United Kingdom (UK) which includes TMJ Medical (formerly Christensen), TMJ Concepts (formerly Techmedica) and Biomet (formerly Lorenz) prostheses. The TMJ Medical (Christensen) prosthesis is metal-on-metal cobalt-chrome alloy with around $10 \%$ exhibiting a foreign body reaction [37]. The long term follow up with this type of replacement was based on the old acrylic on metal model but showed good outcome up to 10 years with failure due to wear of the acrylic component. The TMJ Concepts prosthesis is custom made and has a good success rate reported at 14 years [38,39], but longer in case presentations and company data. This and the Biomet prosthesis are both high molecular weight polyethylene fossa with a cobalt chrome condylar component similar to total knee replacement. Biomet (Lorenz) have a stock and custom prosthesis dependent on whether the stock prosthesis can be made to fit the patient. Patients who undergo TMJ replacement often have had previous surgical interventions thus making them more susceptible for significant postoperative morbidity usually involving the facial nerve. Despite this at one year and beyond function improves by $90 \%$ and pain reduces by $90 \%$, with associated improvements in mouth opening [40].

In UK the National Institute for Health and Clinical Excellence (NICE) has issued guidance on total prosthetic TMJ replacement [41]. These guidelines are comprehensive and much more stringent when compared to guidelines available for other joint replacements. Currently 16 surgeons are involved in TMJ replacement in UK and collectively they have established a TMJ replacement database with the support of BAOMS (The British Association of Oral and Maxillofacial Surgeons). Between 1994 and 2012; 402 patients had 577 joints inserted [42]. Despite the cost and morbidity; studies have provided good evidence that total TMJ replacement has good outcomes in relation to function and pain with few complications of note [40].

\section{Summary}

TMD is a heterogeneous term which encompasses a variety of signs and symptoms. History, clinical examination and appropriate conservative management should precede further investigations as indicated by an appropriate treatment plan to achieve a good overall outcome. Other TMJ pathology should be considered in the differential diagnosis. MRI remains the investigation of choice whilst TMJ arthroscopy should precede more invasive treatment modalities. Open surgery and joint replacement should be reserved for those who have failed less aggressive treatments. Joint replacement is strictly governed by NICE guidelines and should only be carried out by an appropriately trained surgeon in the UK. Overall outcome following TMJ replacement remains good.

\section{References}

1. Hoffmann RG, Kotchen JM, Kotchen TA, Cowley T, Dasgupta M, et al. (2011) Temporomandibular disorders and associated clinical comorbidities. Clin J Pain 27: 268-274.

2. Milam SB (2003) Pathophysiology and epidemiology of TMJ. J Musculoskelet Neuronal Interact 3: 382-390.

3. Poveda Roda, Díaz Fernández JM, Hernández Bazán S, Jiménez Soriano Y, Margaix M, et al. (2008) A review of temporomandibular joint disease (TMJD). Part II: Clinical and radiological semiology. Morbidity processes. Med Oral Patol Oral Cir Bucal 13: E102-109.

4. Ruf S, Pancherz H (1995) Is orthopantomography reliable for TMJ diagnosis? An experimental study on a dry skull. J Orofac Pain 9: 365-374.

5. Crow HC, Parks E, Campbell JH, Stucki DS, Daggy J (2005) The utility of panoramic radiography in temporomandibular joint assessment. Dentomaxillofac Radiol 34: 91-95.

6. Barghan S, Tetradis S, Mallya S (2012) Application of cone beam computed tomography for assessment of the temporomandibular joints. Aust Dent J 57 Suppl 1: 109-118.

7. Westesson PL, Katzberg RW, Tallents RH, Sanchez-Woodworth RE Svensson SA (1987) CT and MR of the temporomandibular joint: comparison with autopsy specimens. AJR Am J Roentgenol 148: 1165-1171.

8. Boeddinghaus R, Whyte A (2013) Computed tomography of the temporomandibular joint. J Med Imaging Radiat Oncol 57: 448-454.

9. Larheim TA (2005) Role of magnetic resonance imaging in the clinical diagnosis of the temporomandibular joint. Cells Tissues Organs 180: 6-21.

10. Lamot U, Strojan P, A urlan PopoviÄ K (2013) Magnetic resonance imaging of temporomandibular joint dysfunction-correlation with clinical symptoms, age, and gender. Oral Surg Oral Med Oral Pathol Oral Radiol 116: 258-263.

11. Shen P, Huo L1, Zhang SY2, Yang C3, Cai XY1, et al. (2014) Magnetic resonance imaging applied to the diagnosis of perforation of the temporomandibular joint. J Craniomaxillofac Surg .

12. Ebrahim S, Montoya L, Busse JW, Carrasco-Labra A, Guyatt GH; Medically Unexplained Syndromes Research Group (2012) The effectiveness of splint therapy in patients with temporomandibular disorders: a systematic review and meta-analysis. J Am Dent Assoc 143: 847-857.

13. Al-Ani MZ, Davies SJ, Gray RJ, Sloan P, Glenny AM (2004) Stabilisation splint therapy for temporomandibular pain dysfunction syndrome. Cochrane Database Syst Rev : CD002778.

14. Sidebottom AJ (2009) Current thinking in temporomandibular joint management. Br J Oral Maxillofac Surg 47: 91-94.

15. Koh H, Robinson PG (2003) Occlusal adjustment for treating and preventing temporomandibular joint disorders. Cochrane Database Syst Rev : CD003812.

16. Mujakperuo HR, Watson M, Morrison R, Macfarlane TV (2010) Pharmacological interventions for pain in patients with temporomandibular disorders. Cochrane Database Syst Rev : CD004715.

17. Moraes Ada R, Sanches ML, Ribeiro EC, Guimarães AS (2013) Therapeutic exercises for the control of temporomandibular disorders. Dental Press J Orthod 18: 134-139.

18. De Riu G, Stimolo M, Meloni SM, Soma D, Pisano M, et al. (2013) Arthrocentesis and temporomandibular joint disorders: clinical and radiological results of a prospective study. Int J Dent 2013: 790648.

19. Manfredini D, Rancitelli D, Ferronato G, Guarda-Nardini L (2012) Arthrocentesis with or without additional drugs in temporomandibular joint inflammatory-degenerative disease: comparison of six treatment protocols*. J Oral Rehabil 39: 245-251.

20. Manfredini D, Piccotti F, Guarda-Nardini L (2010) Hyaluronic acid in the treatment of TMJ disorders: a systematic review of the literature. Cranio 28: 166-176. 
21. Gencer Z, OzkiriÅŸ M, Okur A, Korkmaz M, Saydam L (2014) A comparative study on the impact of intra-articular injections of hyaluronic acid, tenoxicam and betametazon on the relief of temporomandibular joint disorder complaints. J Craniomaxillofac Surg .

22. El-Hakim IE, Elyamani AO. Preliminary evaluation of histological changes found in a mechanical arthropatic temporomandibular joint (TMJ) exposed to an intra-articular Hyaluronic acid (HA) injection, in a rat model. J Craniomaxillofac Surg. 2011; 39:610-4.

23. Schwartz M, Freund B (2002) Treatment of temporomandibular disorders with botulinum toxin. Clin J Pain 18: S198-203.

24. Plesh O, Curtis D, Levine J, McCall WD Jr (2000) Amitriptyline treatment of chronic pain in patients with temporomandibular disorders. J Oral Rehabil 27: 834-841.

25. Zhou H, Hu K, Ding Y (2014) Modified dextrose prolotherapy for recurrent temporomandibular joint dislocation. Br J Oral Maxillofac Surg 52: 63-66.

26. Refai H, Altahhan O, Elsharkawy R. The efficacy of dextrose prolotherapy for temporomandibular joint hypermobility: a preliminary prospective, randomized, double-blind, placebo-controlled clinical trial. J Oral Maxillofac Surg. 2011; 69:2962-70.

27. Hakala RV (2005) Prolotherapy (proliferation therapy) in the treatment of TMD. Cranio 23: 283-288.

28. Weedon S, Ahmed N, Sidebottom AJ (2013) Prospective assessment of outcomes following disposable arthroscopy of the temporomandibular joint. Br J Oral Maxillofac Surg 51: 625-629.

29. Fridrich KL, Wise JM, Zeitler DL (1996) Prospective comparison of arthroscopy and arthrocentesis for temporomandibular joint disorders. J Oral Maxillofac Surg 54: 816-820.

30. Ahmed N, Sidebottom A, O'Connor M, Kerr HL (2012) Prospective outcome assessment of the therapeutic benefits of arthroscopy and arthrocentesis of the temporomandibular joint. Br J Oral Maxillofac Surg 50: 745-748.

31. Rigon M, Pereira LM, Bortoluzzi MC, Loguercio AD, Ramos AL, et al. (2011) Arthroscopy for temporomandibular disorders. Cochrane Database Syst Rev : CD006385.
32. Undt G, Murakami K, Rasse M, Ewers R. Open versus arthroscopic surgery for internal derangement of the temporomandibular joint: a retrospective study comparing two centres' results using the Jaw Pain and Function Questionnaire. J Craniomaxillofac Surg. 2006; 34:234-241.

33. Klüppel LE, Olate S, Serena-Gomez E, De Moraes M, Fernandes-Moreira RW (2010) Efficacy of eminectomy in the treatment of prolonged mandibular dislocation. Med Oral Patol Oral Cir Bucal 15: e891-894.

34. Tzanidakis K, Sidebottom AJ (2013) Outcomes of open temporomandibular joint surgery following failure to improve after arthroscopy: is there an algorithm for success? Br J Oral Maxillofac Surg 51: 818-821.

35. Holmlund A, Lund B, Weiner CK (2013) Discectomy without replacement for the treatment of painful reciprocal clicking or catching and chronic closed lock of the temporomandibular joint: a clinical follow-up audit. Br J Oral Maxillofac Surg 51: e211-214.

36. Dimitroulis G (2011) A critical review of interpositional grafts following temporomandibular joint discectomy with an overview of the dermis-fat graft. Int J Oral Maxillofac Surg 40: 561-568.

37. Sidebottom AJ, Speculand B, Hensher R (2008) Foreign body response around total prosthetic metal-on-metal replacements of the temporomandibular joint in the UK. Br J Oral Maxillofac Surg 46: 288-292.

38. Speculand B. Current status of replacement of the temporomandibular joint in the United Kingdom Br J Oral Maxillofac Surg. 2009; 47:37-41.

39. Mercuri LG, Edibam NR, Giobbie-Hurder A (2007) Fourteen-year follow-up of a patient-fitted total temporomandibular joint reconstruction system. J Oral Maxillofac Surg 65: 1140-1148.

40. Sidebottom AJ, Gruber E (2013) One-year prospective outcome analysis and complications following total replacement of the temporomandibular joint with the TMJ Concepts system. Br J Oral Maxillofac Surg 51: 620-624.

41. http://guidance.nice.org.uk/IPG329

42. Idle MR, Lowe D, Rogers SN, Sidebottom AJ, Speculand B, et al. (2014) UK temporomandibular joint replacement database: report on baseline data. Br J Oral Maxillofac Surg 52: 203-207. 